Article

\title{
Preparation and Properties of Minocycline-Loaded Carboxymethyl Chitosan Gel/Alginate Nonwovens Composite Wound Dressings
}

\author{
Yingjun Gao, Xing Zhang and Xiangyu Jin * (1) \\ Key Laboratory of Textile Science and Technology of the Ministry of Education, College of Textiles, \\ Donghua University, Shanghai 201620, China; gaoyingj@hotmail.com (Y.G.); tulip_90@163.com (X.Z.) \\ * Correspondence: jinxy@dhu.edu.cn; Tel.: +86-021-67792787
}

Received: 9 September 2019; Accepted: 7 October 2019; Published: 11 October 2019

check for updates

\begin{abstract}
As derivatives from marine natural biomaterials, alginate-based and chitosan-based biomaterials are commonly used in wound dressings. Calcium alginate fiber (CAF) dressings possess excellent absorption and unique gel forming performance, but the low bioactivity limits its application in wound healing. Carboxymethyl chitosan (CM-Chit) has excellent antibacterial activity, but the gel structure with weak mechanical properties restricts its application. In this study, minocycline (Mino)/CM-Chit solution was coated on the surface of plasma treated CAF needle-punched nonwovens, and then Mino loaded CM-Chit gel/CAF nonwovens composite dressings were fabricated by EDC/NHS (1-3-(3-dimethylaminopropyl)-3-ethylcarbodiimide hydrochloride/N-hydroxysuccinimide) crosslinking. The dressings had a porous composite structure, which allowed them to quickly absorb and store a large number of wound exudates. Skin-like tensile performance allowed the dressings to provide a better healing environment. Antibacterial assay against Escherichia coli and Staphylococcus aureus indicated that the addition of Mino significantly improved the antibacterial activity of the wound dressings. The tight structure of CM-Chit gel prevented the burst release of Mino so that the dressings had antibacterial activity in a certain period of release time. Cell culture assay showed that the dressings had excellent cell biocompatibility. As new functional dressings, the prepared composite dressings had excellent potential in the clinical healing of wounds.
\end{abstract}

Keywords: alginate fibers; carboxymethyl chitosan; wound dressings; antibacterial activity; drug release

\section{Introduction}

As the skin substitute, the ideal wound dressings should possess the following functions: (1) be a barrier to protect wound from contamination by chemicals and microorganisms; (2) allow air exchange, which provides oxygen for cell growth and excreting carbon dioxide produced by cells; (3) remove wound exudates in time and maintain the moist microenvironment between wound, wound exudates and dressings; (4) be non-toxic and non-allergenic to wound [1-4]. Nowadays, the preparation of new generation functional dressings with biological activity has become a hot research topic. These functional dressings possess various bioactivities, such as rapid hemostasis, antibacterial, anti-inflammatory, promoting cell proliferation, tissue regeneration and wound healing. The strategies of producing these functional dressings can be classified into two categories. The first is to prepare dressings directly from biologically active materials such as collagen, chitosan and alginate. The second is to add bioactive macromolecules or small molecules into the dressings, such as proteins, growth factors, drugs and even cells [5-8].

Chitosan is a cationic marine natural polysaccharide obtained from chitin after deacetylation of the $\mathrm{N}$-acetyl glucosamine groups. The chemical structure of chitosan is composed by D-glucosamine 
and N-acetyl-D-glucosamine by $\beta$ (1-4) glycosidic linkages [9]. Owing to its excellent biocompatibility and biodegradability, chitosan-based biomaterials are wildly used in biomedical fields, such as tissue engineering, drug delivery, tissue regeneration and wound healing [10-15]. The primary amine groups endow chitosan unique properties such as in situ gelation, permeation enhancement, cationic nature and antibacterial. Previous researches indicate that chitosan can inhibit the growth of bacteria and fungi and possess broad-spectrum antibiotic activity, high bacteriostatic rate. Meanwhile, chitosan has hemostatic effects and can promote the activation of macrophages and cytokines [16,17]. In the early phase of wound healing, chitosan can induce collagen synthesis and accelerate the re-epithelialization, the angiogenesis of the granular layer regeneration, which is positive for wound healing $[18,19]$. The application of chitosan in functional dressings has attracted increasing attention due to its antimicrobial activity and healing-promoting performance [20-22]. Chitosan has low solubility in water and common organic solvents, so some reagents need to be added in the preparation of chitosan-based biomaterials. Some of the reagents are bio-toxic, which limits the application of chitosan-based biomaterials in biomedicine. Carboxymethyl chitosan is a water-soluble derivative of chitosan. According to the different substitution sites of carboxymethyl groups, carboxymethyl chitosan can be divided into three types: $\mathrm{N}, \mathrm{O}$-carboxymethylated chitosan, $\mathrm{O}$-carboxymethylated chitosan and $\mathrm{N}$-carboxymethylated chitosan. Carboxymethyl chitosan biomaterials can be fabricated in neutral water medium, thus maintaining the activity of active substances such as small molecule drugs, proteins and cells, which expands the application range of chitosan [23-26]. Meanwhile, carboxymethyl chitosan possesses better sustained and controlled drug release performance [27-30]. Compared with chitosan, carboxymethyl chitosan has better biocompatibility, high moisture retention ability and enhanced antimicrobial property so it has better application in wound healing. The antimicrobial properties of N, O-carboxymethyl chitosan and O-carboxymethyl chitosan are better than that of chitosan. Carboxymethyl chitosan has excellent bioactivity, biodegradability, antimicrobial activity and biological affinity. It is widely used in drug delivery systems, antimicrobial dressings, tissue engineering scaffolds [31-34].

Sodium Alginate is a linear marine natural polysaccharide composed of $\beta$-D-mannuronate (M) and $\alpha$-D-guluronate $(\mathrm{G})$ by 1, 4-glycosidic covalent linkages. These two monomers are combined in different amount and sequential distribution to form various molecular chain fragments, such as M-blocks (MMM) G-blocks (GGG) and G-M-blocks (GMGMG) [35]. Sodium alginate possesses unique multivalent cationic chelating property. In the presence of $\mathrm{Ca}^{2+}$ ions, sodium alginate is synthesized into insoluble calcium alginate with "egg-box" macromolecular structure [36,37]. Thus, the materials with different structures and properties, such as micro- and nano- microfibers, hydrogels, films etc. can be produced in easy crosslinking methods. Due to the excellent biocompatibility and mechanical properties, calcium alginate fibers and its textile assembly with "tissue-like" 3D structure are extensively used in biomedical applications, such as tissue engineering scaffold, drug carriers, wound dressings [38-42]. The $\mathrm{Ca}^{2+}$ ions chelated with $\mathrm{M}$ groups are easily replaced by $\mathrm{Na}^{+}$ions in wound exudates and then form sodium alginate hydrogels, producing moist healing conditions which are proved to reduce wound healing times [1]. The hydrogels reduce the pain severity and can be easily removed from wound without extra trauma [43]. The alginate fiber dressings are widely used in the treatment of moist chronic wounds such as diabetic foot ulcers, toxic epidermal necrolysis, necrotic leg ulcers, etc. [2,44,45]. Calcium alginate fiber dressings have limited biological activity and do not add active substances such as small molecule drugs, proteins, growth factors, which reduces its healing performance and application in wound healing.

Alginate has no ability to promote cell proliferation, migration, differentiation and specific gene expression. Meanwhile, the weak mechanical performance makes it difficult for carboxymethyl chitosan to produce a scaffold structure. In order to overcome the limitation of individual biomaterials and prepare biopolymers with multiple functions, hydrogels, microfibers, membranes and films composed by alginate and carboxymethyl chitosan have been prepared and their potential applications as wound dressings in promoting wound healing have been investigated. Compared with 
dressings made of individual biopolymer, these composite dressings possess better healing-promoting performance $[26,46,47]$. These biomaterials have excellent bioactivity, but their structures are mostly hydrogels or gels. The mechanical properties of hydrogels and gels are quite different from those of tissues and organs, which limits their application in biomedical fields, especially in wound dressings.

In this study, we developed the composite dressings made of carboxymethyl chitosan (CM-Chit) gel and calcium alginate fiber (CAF) needle-punched nonwovens. The mechanical performance, the physicochemical properties, the cell compatibility and the antibacterial activity were characterized. Meanwhile, the drug-embedded carboxymethyl chitosan-alginate composite dressings were prepared to explore the potential of the composite dressings as a drug delivery system, which endowed the composite dressings with more functions. The results evidenced that the composite dressings could be used as functional compress to accelerate wound healing.

\section{Results and Discussion}

\subsection{Fabrication of CM-Chit Gel/CAF Nonwovens Composite Wound Dressings}

As shown in Figure 1A, CA fibers had a certain crimp, which improved the entanglement and friction between fibers. After opening, carding and lapping, CA fibers intertwined and formed webs with a fluffy 3D structure. CA fibers were driven and moved along the thickness direction, forming fiber bundles with vertical distribution. The fiber webs were reinforced by the fiber bundles to form needle-punched nonwovens with excellent mechanical properties and porous 3D structure. As shown in Figure 1B, in the chemical crosslinking formation reaction of CM-Chit gel, EDC and NHS participated in only as intermediates but not as part of the crosslinked products. In the reaction conditions of ethanol, the $\mathrm{O}$-isoacylurea was formed by coupling the EDC and carboxyl groups in CM-Chit macromolecular chains, and then, the amide bond was formed between the $O$-isoacylurea and the amino groups in the CM-Chit macromolecular chains, making the macromolecular chains crosslinked. The existence of NHS enhanced the stability of the crosslinked products and the residual EDC/NHS was removed after washing with Milli-Q water to reduce the biological toxicity of the composite wound dressings. As shown in Figure 1C, CM-Chit macromolecular chains distributed randomly and entangled with each other to form the solution with a certain viscosity. After chemical crosslinking reaction, the entangled macromolecular chains formed chemical bonds through amide bonds, which enhanced the interaction between macromolecular chains to form gel with stable structure.

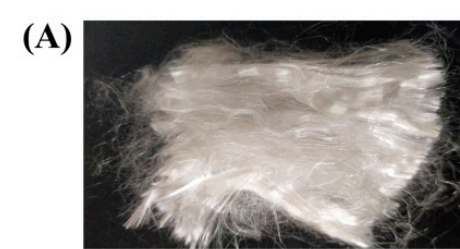

CAF

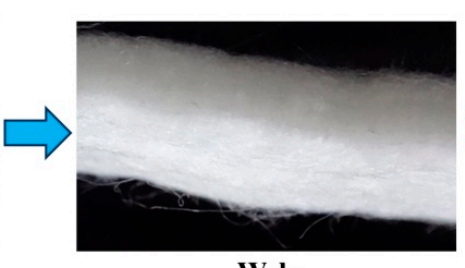

Webs

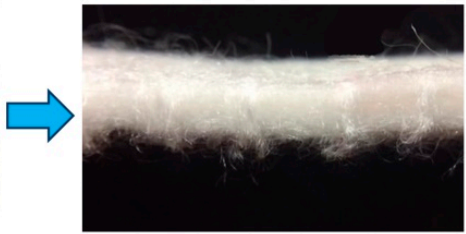

CAF Nonwovens
(B)

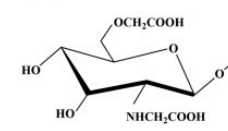

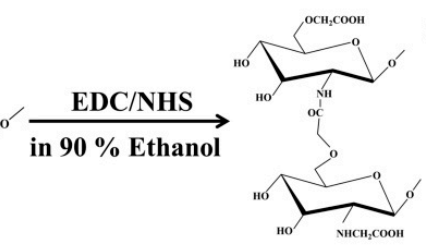

(C)

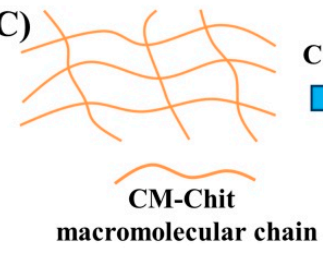

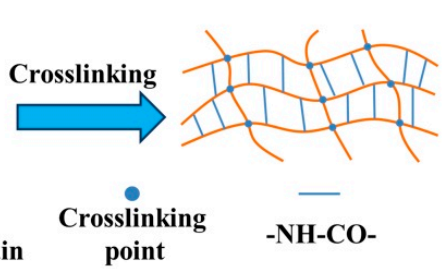

Figure 1. Structure changes in the fabrication of composite wound dressings. (A) The structure of CAF, webs and needle-punched nonwovens. (B) The chemical structure and (C) the macromolecular chains structure of CM-Chit during crosslinking. 


\subsection{Morphology and Structure Characterization}

\subsubsection{The Rheological Behaviors and Diffusion of CM-Chit Solution}

In the preparation of the composite wound dressings, the performance of solution, especially the rheological properties, greatly affects the structure and properties of dressings. The rheological properties of CM-Chit solution were shown in Figure 2A. As the CM-Chit concentration increased from $1 \%(w / v)$ to $4 \%(w / v)$, the solution viscosity improved from 0.05 PA s to $1.20 \mathrm{PA}$ s. With the increase of macromolecules content in solution, the entanglement degree and interaction force between macromolecular chains increased. Especially when the solution concentration increased from $3 \%(w / v)$ to $4 \%(w / v)$, the solution viscosity increased greatly. When solution was sheared, the entanglement between macromolecular chains was destroyed, reducing the viscosity of the solution. With the increase of the shear rate, the degree of disruption of intermolecular entanglement increased. The effect of shear rate on the viscosity of solution with high concentrations solution was significantly higher than that of solution with low concentrations. When the shear rate was higher than $20 \mathrm{r} / \mathrm{s}$, the viscosity of solution with high concentrations decreased obviously and tended to be stable. The surface tension was another important indicator of solution properties, which was closely related to the density and viscosity of solution. As shown in Figure 2B, with the increase of solution concentration and viscosity, the surface tension of CM-Chit solution increased significantly. The large surface tension of the solution with high concentrations improved the force needed to surmount the spontaneous contraction, which significantly reduced the fluidity of CM-Chit solution and the diffusion of the solution to CAF nonwovens. As shown in Figure 2C, when the CM-Chit solution concentration was low, the tight composite structure reduced the thickness of the composite wound dressings. The high fluidity and viscosity of the solution with low concentrations promoted the diffusion of the solution into the nonwovens, making the gel diffusion depth reach $70 \%$ of the thickness of the composite dressings. With the increase of concentration, the diffusion depth of CM-Chit solution into CAF nonwovens declined, which made the thickness of CM-Chit gel increase and the thickness of the composite dressings decline significantly.

\subsubsection{The Cross-Section Morphology and Structure Characterization}

The optical cross-section morphology of the composite wound dressings was shown in Figure 2D. The composite wound dressings possessed porous structure composed of gel and nonwovens. The porous structure was divided into three parts: CM-Chit gel, gel/nonwovens composite and nonwovens. As the diffusion of CM-Chit solution decreased, the boundary between gel and nonwovens became obvious. The cross-section SEM images of the composite dressings (Figure 2E) showed that when the CM-Chit solution concentration was low, the solution penetrated into the CAF nonwovens and diffused along the fibers and the pores in the nonwovens. As the concentration increased, the penetration of CM-Chit solution along the thickness of CAF nonwovens and the diffusion along fibers and pores decreased, which reduced the composite structure and weakened the structural change of CAF nonwovens. 
(A)

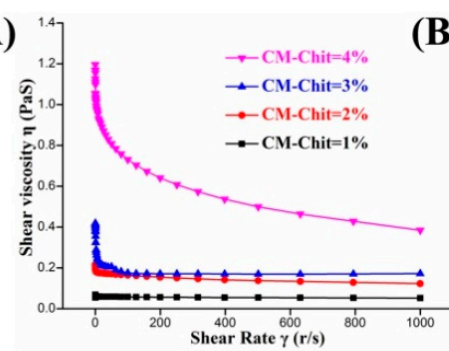

(B)

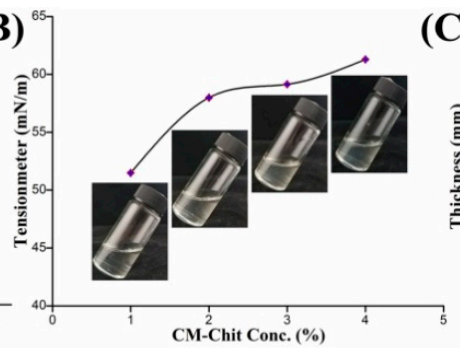

$\left.(C)^{30}\right]$ TaF+CM-Chit

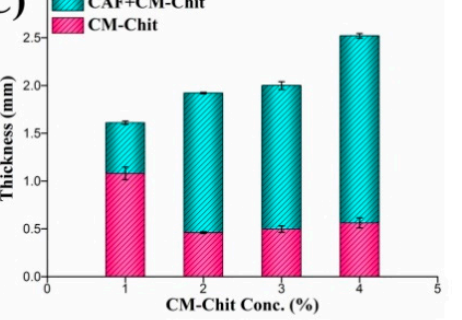

\section{(D)}

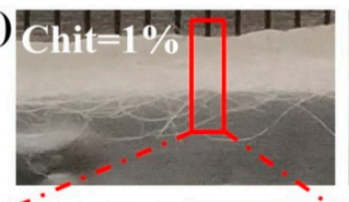

(E)

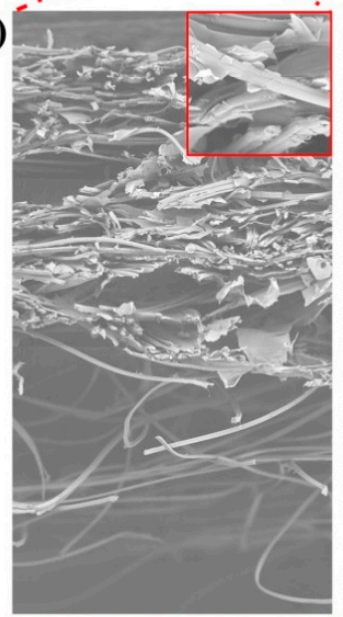

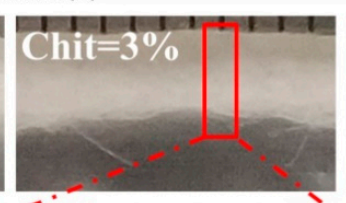

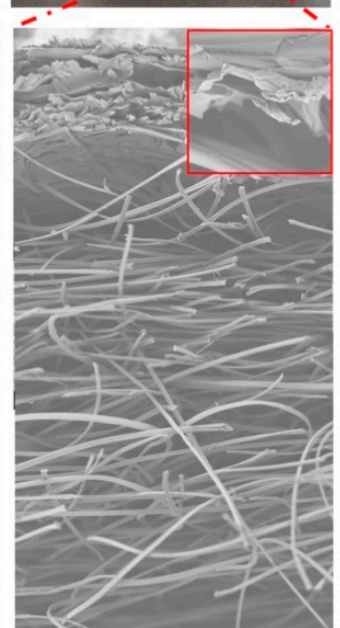

CM-Chit solution

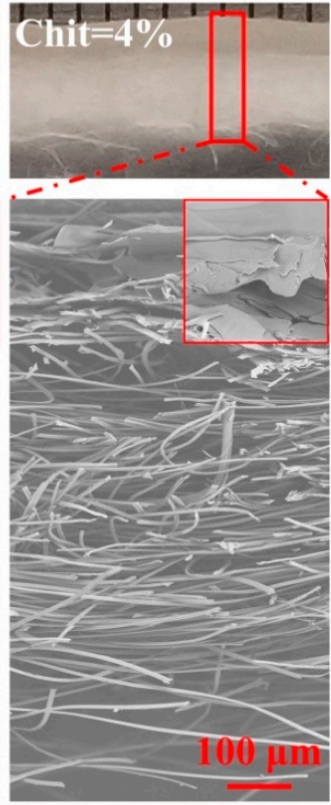

Diffusion depth of CM-Chit solution

Figure 2. (A) The rheological behaviors and (B) the surface tension curves of CM-Chit solution with different concentrations. (C) The thickness of the composite wound dressings and CM-Chit gel. The cross-section morphology of the composite dressings: (D) The optical images and (E) the SEM images.

\subsubsection{The Surface Morphology and Structure Characterization}

As shown in Figure 3A,B, energy dispersive X-ray (EDX) scanning was used to observe the distribution of characteristic elements on the CM-Chit gel surface and CAF nonwovens surface. The distribution of CM-Chit gel was characterized by $\mathrm{N}$ element content, meanwhile the distribution of $\mathrm{CA}$ fibers was characterized by Ca element content. On the surface of CM-Chit gel, a large number of $\mathrm{N}$ elements were distributed, with a content of $9.16 \%$. The content of $\mathrm{Ca}$ was $5.01 \%$, and the relationship between the distribution of $\mathrm{N}$ and $\mathrm{Ca}$ was complementary. On the surface of CAF nonwovens, the $\mathrm{Ca}$ element was evenly distributed with a content of $15.49 \%$. Due to a large number of pores between CA fibers, the $\mathrm{N}$ element distributed on the surface of CAF nonwovens was detected with a relatively low content, which was $1.07 \%$. 


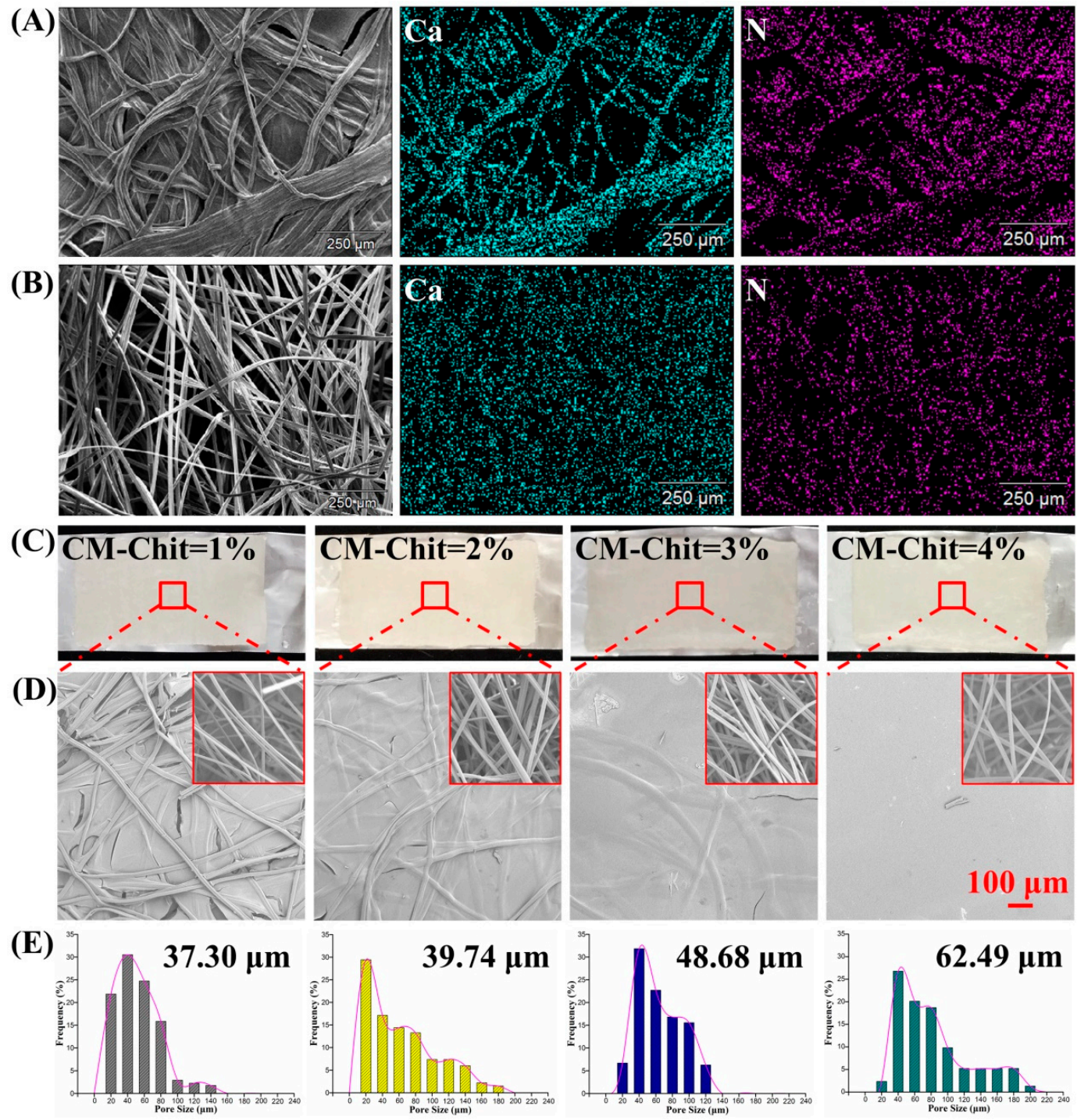

Figure 3. EDX mapping images of (A) the CM-Chit gel surface, (B) the CAF nonwovens surface. The surface morphology of the composite dressings: (C) the optical images, (D) the SEM images. (E) The pore size and its distribution of the composite dressings.

Figure 3C,D showed the surface morphology of the composite dressings. When the amount of CM-Chit solution penetrated into CAF nonwovens was high, the obvious fibrous structure was observed on the CM-Chit gel surface, which separated the CM-Chit gel structure into different regions. When the amount of CM-Chit solution diffused into CAF nonwovens declined, the fibers distributed on the gel surface gradually decreased and eventually disappeared. The CM-Chit gel interacted and formed a compact surface structure.

As shown in Figure 3E, with the increase of CM-Chit solution concentration from 1\% $(w / v)$ to $4 \%$ $(w / v)$, the mean pore size of the composite dressings gradually increased from $37.30 \mu \mathrm{m}$ to $62.49 \mu \mathrm{m}$. The main distribution ranges of pore size also changed significantly. The main distribution range of pore size of the composite dressings prepared by CM-Chit solution with concentrations of $1 \%(w / v)$, $2 \%(w / v), 3 \%(w / v)$ and $4 \%(w / v)$ were $20-60 \mu \mathrm{m}, 20-80 \mu \mathrm{m}, 40-100 \mu \mathrm{m}$ and $40-120 \mu \mathrm{m}$, respectively. With the increase of CM-Chit solution concentration, the number of pores with large size increased significantly. This was mainly because that with the decrease of CM-Chit permeability, the destruction of CAF nonwovens porous structure caused by CM-Chit gel structure was reduced, thus increasing the distribution of large pores in the composite dressings. 


\subsection{The Properties Characterization}

\subsubsection{The Chemical Structure Characterization}

The chemical structure of the composite dressings was characterized by FTIR and XRD spectra. The functional groups structure of the composite dressings before and after crosslinking was shown in Figure 4A. From the FTIR spectra of CM-Chit, the absorption peaks at $3271 \mathrm{~cm}^{-1}, 2910 \mathrm{~cm}^{-1}$ and $1730 \mathrm{~cm}^{-1}$ were the characteristic peaks of $\mathrm{O}-\mathrm{H}, \mathrm{C}-\mathrm{H}$ and $-\mathrm{COOH}$ groups, respectively. The absorption peaks at $1632 \mathrm{~cm}^{-1}, 1555 \mathrm{~cm}^{-1}$ and $1402 \mathrm{~cm}^{-1}$ characterized the COO-, $\mathrm{N}-\mathrm{H}$ and $-\mathrm{CH}_{2} \mathrm{COOH}$ groups, which indicated the transition of amino and hydroxyl groups in chitosan macromolecules caused by carboxymethylation. From the FTIR spectra of the crosslinked CM-Chit, the stretching vibration peaks of $-\mathrm{COOH}$ groups at $1730 \mathrm{~cm}^{-1}$ disappeared, and the absorption peaks of macromolecular chains at $1632 \mathrm{~cm}^{-1}$ and $1402 \mathrm{~cm}^{-1}$ decreased, indicating the decrease of carboxymethyl groups linked with amino groups. The characteristic peaks at $1550 \mathrm{~cm}^{-1}, 1407 \mathrm{~cm}^{-1}$ and $1025 \mathrm{~cm}^{-1}$ moved to $1555 \mathrm{~cm}^{-1}$, $1402 \mathrm{~cm}^{-1}$ and $1023 \mathrm{~cm}^{-1}$, indicating the changes of $\mathrm{N}-\mathrm{H}, \mathrm{C}=\mathrm{O}, \mathrm{C}-\mathrm{N}$ and other groups. With the increase of CM-Chit concentration, the number of macromolecular chains involved in crosslinking increased, and the peak strength of characteristic absorption peaks of - $\mathrm{COOH}$ group decreased gradually; meanwhile, the peak strength of characteristic absorption peaks of $\mathrm{N}-\mathrm{H}$ and $\mathrm{C}-\mathrm{H}$ groups increased gradually. After crosslinking, the interconnected macromolecular chains formed a compact aggregated structure. With the increase of CM-Chit concentration, the degree of entanglement and crosslinking between macromolecular chains increased, resulting in a significant increase in the crystal structure and the intensity of the diffraction peaks at $2 \theta=13.282^{\circ}$ and $2 \theta=38.428^{\circ}$. The crystallinities of the composite dressings fabricated by CM-Chit solution with concentration of $1 \%(w / v), 2 \%(w / v), 3 \%(w / v)$ and $4 \%$ $(w / v)$ were calculated as $21.96 \%, 23.98 \%, 26.16 \%$ and $34.27 \%$, respectively.
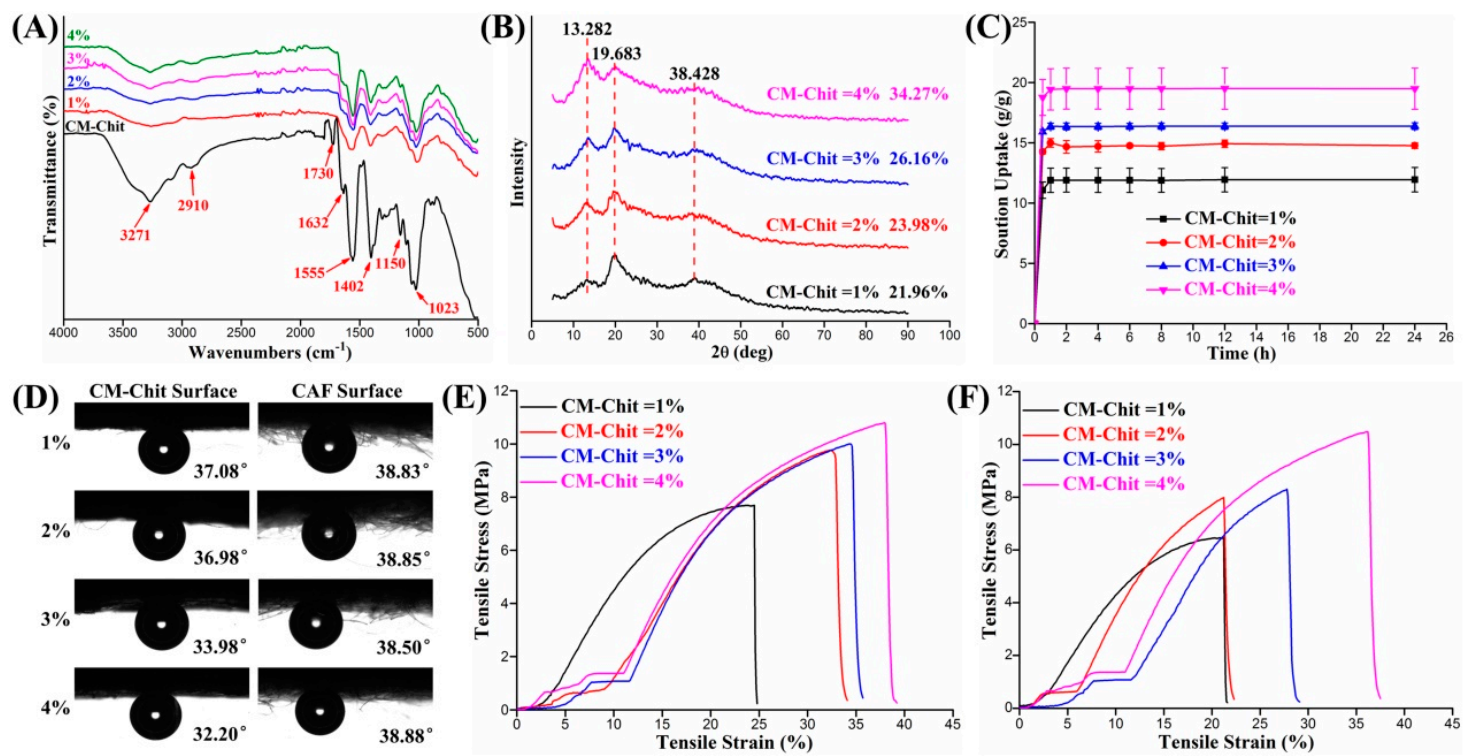

Figure 4. Characterization of physicochemical properties of the composite wound dressings: (A) The FTIR spectrum. (B) The XRD spectrum. (C) The solution uptake. (D) The contact angels. (E) The strain-stress curves in MD. (F) The strain-stress curves in CD.

\subsubsection{The Hydrophilic Properties Characterization}

The solution absorption performance of the composite dressings was shown in Figure $4 \mathrm{C}$. The composite dressings absorbed solution quickly and reached the maximum uptake ratio in a short time and kept stable. With the increase of CM-Chit solution concentration, the solution absorption of the composite dressings increased significantly. CM-Chit gel and CA fibers contain a large number of polar groups, which makes them have excellent absorption performance [33,35]. Owing to the porous 
structure of the composite dressings, the solution absorbed by the composite dressings was divided into four parts: the solution absorbed by CM-Chit gel, the solution absorbed by CA fiber, the solution distributed in the composite structure and the solution distributed in the pores of CAF nonwoven. When the CM-Chit concentration was low, the high proportion of CM-Chit gel encapsulated the fibers, reducing the amount of solution absorbed by the pores of CAF nonwovens. The solution mainly distributed in the CM-Chit gel and fibers; meanwhile, the smaller pore size and porosity resulted in lower solution absorption and storage capacity. For the composite dressings fabricated by CM-Chit with high concentration, the large pore size and high porosity proportion improved the solution absorption and storage properties of the composite dressings.

\subsubsection{The Contact Angles}

The contact angles of the surface of CM-Chit gel and CAF nonwovens were illustrated in Figure 4D. For hydrophilic materials, the contact angle decreases with the increase of absorbency $[48,49]$. The contact angle of CM-Chit gel surface was smaller than that of CAF nonwovens surface, indicating that the CM-Chit gel possessed better hydrophilicity. The increase of CM-Chit concentration significantly improved the hydrophilicity of the gel. When the concentration increased from $1 \%(w / v)$ to $4 \%(w / v)$, $\mathrm{CM}$-Chit solution crosslinked and formed a homogeneous gel surface without CA fibers distribution, reducing the contact angle from $37.08^{\circ}$ to $32.20^{\circ}$. Due to the structure of CAF, nonwovens surface was stable, and the contact angle of CAF nonwovens did not change with the increase of CM-Chit solution concentration.

\subsubsection{Tensile Mechanical Properties Testing}

The tensile properties of the composite dressings were shown in Figure 4E,F. The tensile strengths of the composite dressings fabricated by $\mathrm{CM}$-Chit solution with different concentrations were in the range of 6-12 MPa with strain range of $20 \%-40 \%$. It was in the range of tensile mechanical properties of healthy skin tissue (tensile strength: 1-20 MPa, tensile strain: 30\%-70\%) [50]. Therefore, the composite dressings had excellent biomechanical compliance to use as a substitute for healthy skin in the clinical treatment of wounds. Compared with the tensile curves of nonwovens, the tensile process of the composite dressings was more complicated due to the existence of composite structure, which was divided into three stages. The first stage was the CM-Chit gel stretching stage. At this stage, the gel was stretched along the tensile direction, and the interaction between macromolecule chains was the main force. The second stage was the straightening stage of CA fibers. At this stage, the CM-Chit gel fractured and the interactions between fibers were the main force. With the rearrangement and stretching of a large amount of fibers, the tensile strength of the composite dressings increased significantly. The third stage was the stretching stage of CAF nonwovens, in which the entanglement force, cohesion force and friction force between CAF fibers were the main mechanical action forms. With the increase of CM-Chit concentration, tighter entanglement and crystalline structure of macromolecule chains improved the tensile strength and strain of the gel at the first stage. The increase of the proportion of CAF nonwovens enhanced the tensile mechanical performance of the composite dressings fabricated with high CM-Chit concentration.

\subsection{Drug Release and in Vitro Antibacterial Activity of Mino-Loaded Composite Dressings}

\subsubsection{The Loading and Release of Mino}

The absorbance of Mino-PBS solutions with concentrations of 100, 50, 25, 12.5, 6.25, 3.125 and $1.5625 \mathrm{ug} / \mathrm{mL}$ at $272 \mathrm{~nm}$ was measured by ultraviolet spectrophotometer. The standard curve of Mino concentration ( $\mathrm{C}$ as $\mathrm{y}$ ) and absorbance ( $\mathrm{A}$ as $\mathrm{x}$ ) was drawn and the standard equation was calculated (shown in Figure 5A). The standard absorption equation of Mino was $\mathrm{A}=0.06862 \mathrm{C}$. As shown in Figure $5 \mathrm{~B}$, when the concentration of CM-Chit solution increased from $1 \%(w / v)$ to $4 \%(w / v)$, the Mino 
loading rate of $\mathrm{CM}-\mathrm{Chit}$ gel increased from $53 \%$ to $79.5 \%$, indicating that tight macromolecular chains entanglement structure facilitated the drug loading.
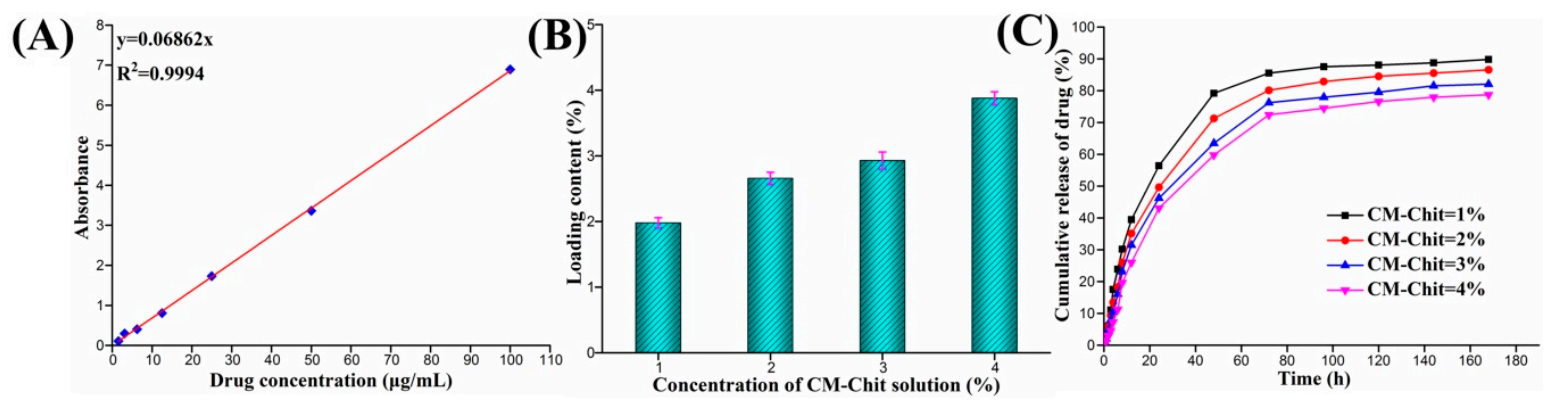

Figure 5. Drug loading and release assay: (A) Standard curve the drug concentration. (B) The drug loading content, $(\mathbf{C})$ the drug release curves of CM-Chit gel with different concentrations.

The Mino release profile from the Mino loaded composite dressings was investigated and the release curves were illustrated in Figure 5C. In the initial $4 \mathrm{~h}$, the cumulative release amounts of CM-Chit gel with different concentrations were $17.5 \%, 13.5 \%, 10 \%$ and $7 \%$, respectively, which indicated that CM-Chit gel avoided drug burst release. Subsequent to the initial fast drug release, the drug release became sustained and gentle. When the release time was $168 \mathrm{~h}$, the cumulative drug release amounts were $90 \%, 86.5 \%, 82 \%$ and $79 \%$, respectively. The concentration of CM-Chit slightly affected the drug release profile loaded in the composite dressings. At the same release time, the cumulative drug release amount declined with the increase of $\mathrm{CM}$-Chit concentration. The drug release from the composite dressings fabricated by low $\mathrm{CM}$-Chit concentration reached the dynamic equilibrium quickly. For the composite dressings fabricated by CM-Chit of a concentration of $4 \%(w / v)$, the drug release reached the dynamic balance at $120 \mathrm{~h}$. The high $\mathrm{CM}$-Chit concentration and tight gel network made it difficult for the loaded drugs to penetrate into the solution, thus prolonging the time of drug release and the effective drug concentration.

\subsubsection{In Vitro Antibacterial Activity Assay}

The loaded Mino was expected to improve the antimicrobial activity of the composite dressings. The antibacterial activity of CAF nonwovens, composite dressings and Mino loaded composite dressings were evaluated by inhibition zone after $24 \mathrm{~h}$ of bacterial culture. As shown in Figure 6A,B, CAF nonwovens had no antimicrobial activity and did not inhibit the apparent growth of Escherichia coli (E. coli) and Staphylococcus aureus (S. aureus). The positive charge in CM-Chit macromolecules interacts with the negative charge on the surface of bacteria, destroying the cell membrane of bacteria and causing cell death [31]. The widths of inhibition zones of the composite dressings against $E$. coli and S. aureus were $0.95 \mathrm{~mm}$ and $1.2 \mathrm{~mm}$, respectively. Therefore, the composite dressings possessed slight antimicrobial activity against $E$. coli and S. aureus. When the composite dressings contained Mino with a low concentration of $2.5 \%(w / v)$, the widths of inhibition zones against $E$. coli and $S$. aureus expanded to $13.82 \mathrm{~mm}$ and $11.45 \mathrm{~mm}$, respectively. The antimicrobial activity of the composite dressings was significantly improved by the loading of Mino, meanwhile the width of inhibition zone against $S$. aureus expanded to $18.8 \mathrm{~mm}$ with the Mino concentration increased to $10 \%(w / v)$. 
(A)

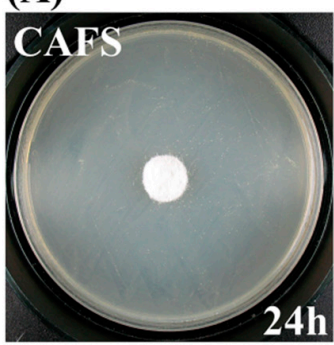

(B)
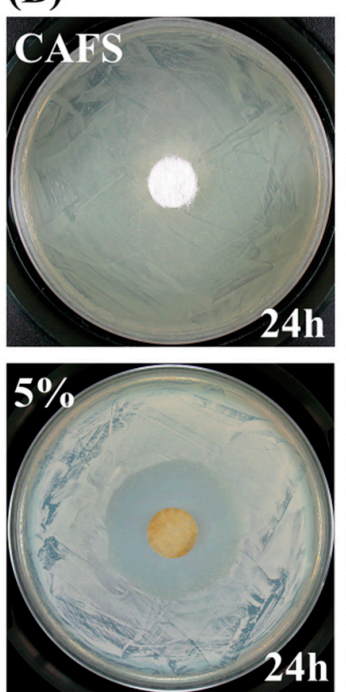

(C)

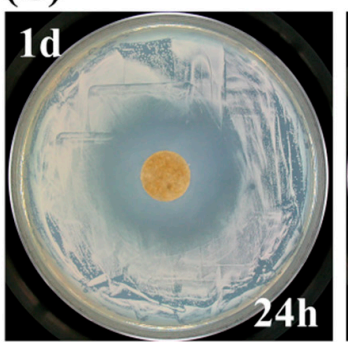

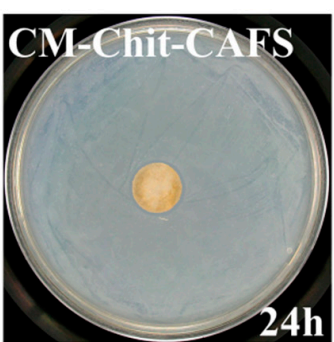
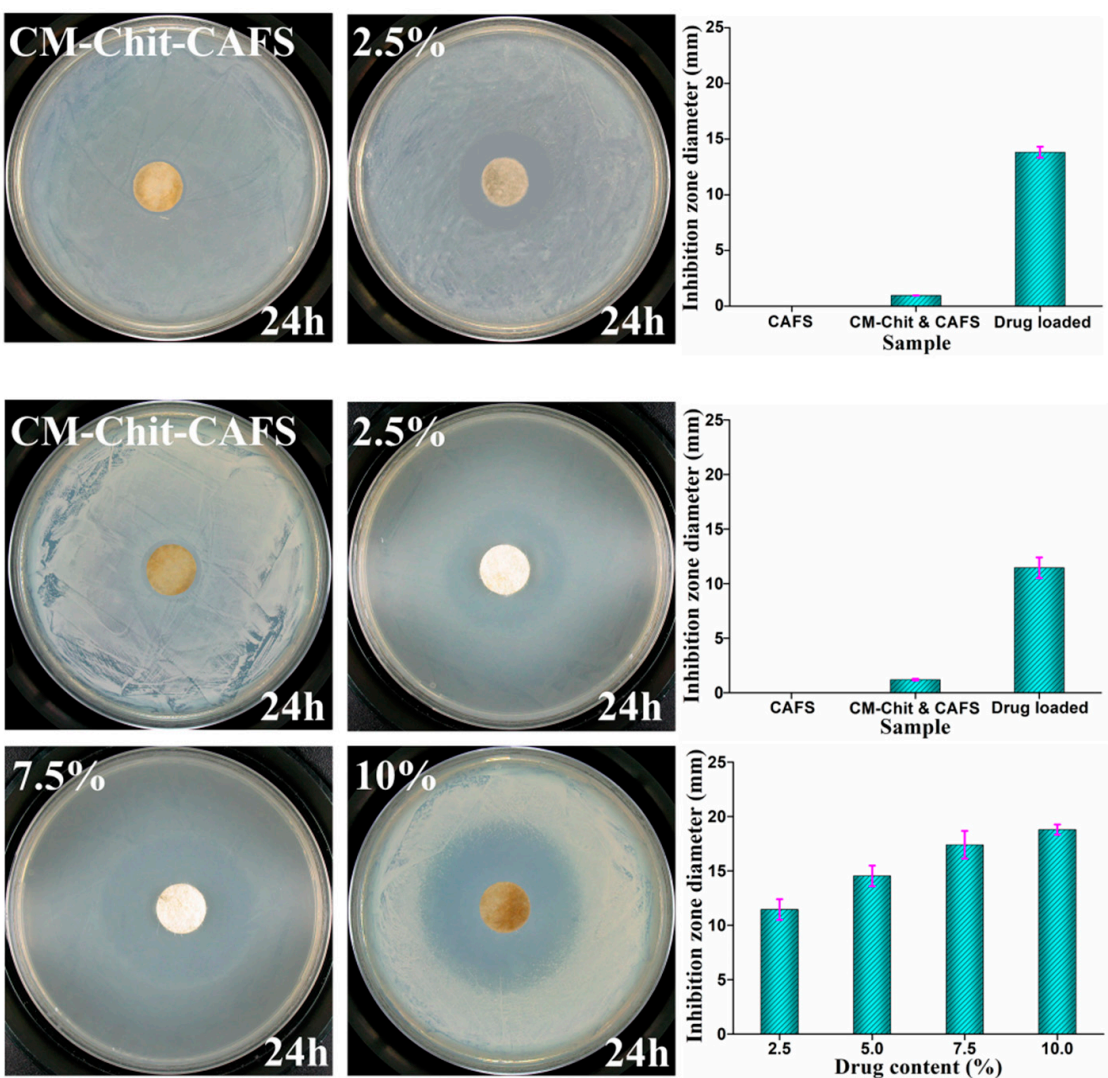

$4 h$
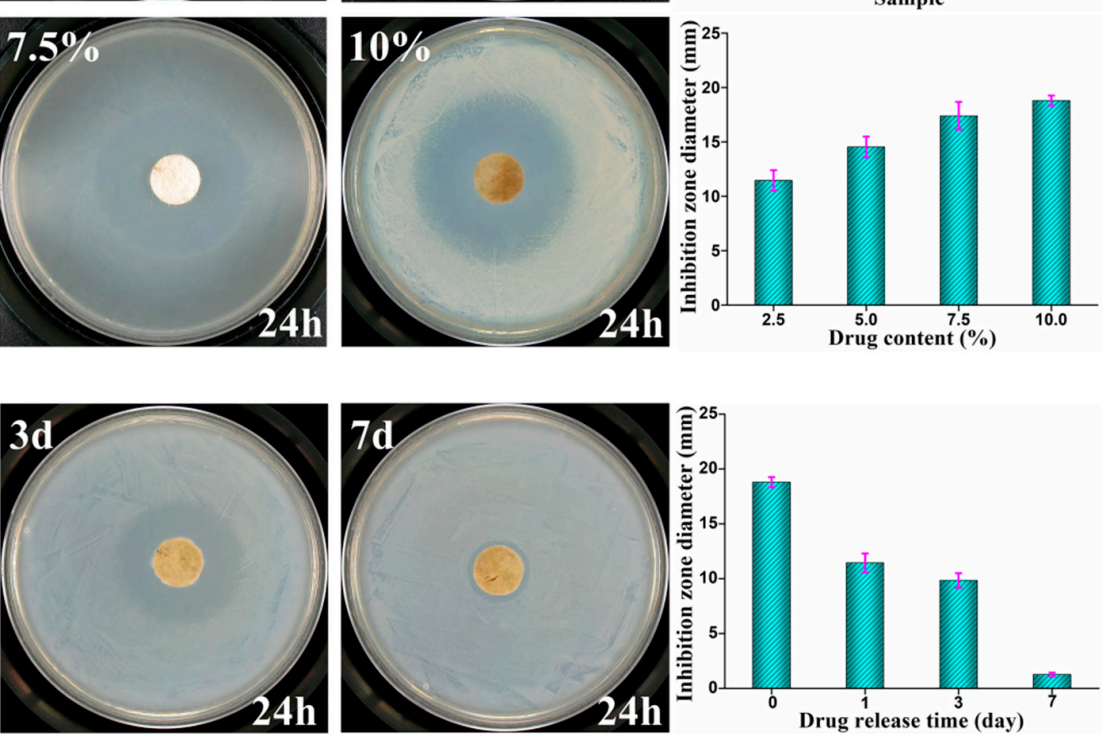

Figure 6. The antibacterial activity of $\mathrm{CAF}$ nonwovens, the composite dressings and the Mino loaded composite wound dressings (A) against $E$. coli (B) against $S$. aureus. (C) The antibacterial activity of Mino loaded composite dressings released for 1,3,7 days against $S$. aureus. 
The composite dressings loaded with 10\% (w/v) Mino were immersed in PBS to release. The antibacterial activity against $S$. aureus of the Mino loaded composite dressings ( $4 \%(w / v)$ CM-Chit, $10 \%$ $(w / v)$ Mino) after 1, 3, 7 days release was investigated and illustrated in Figure 6C. With the release of drug, the width of the inhibition zone and the antimicrobial activity of Mino loaded composite dressings decreased gradually. The widths of the inhibition zone of the Mino loaded composite dressings were $11.84 \mathrm{~mm}$ and $9.84 \mathrm{~mm}$, respectively after 1 and 3 days of release, indicating that the Mino loaded composite dressings still had obvious antibacterial activity. After 7 days of release, the inhibition zone of the drug loaded composite dressings was only $6.75 \%$ of the inhibition zone of the unreleased Mino loaded composite dressings, but its antibacterial activity was still better than those of the composite dressings and CAF nonwovens. The results indicated that Mino in the composite dressings maintained certain antimicrobial activity and effective antimicrobial concentration in a certain period of time, which inhibited the growth of bacteria.

\subsection{HUVECs Proliferation and Spreading on the Composite Dressings}

The cell cytotoxicity of CAF nonwovens, composite dressings and Mino loaded composite dressings was evaluated by studying the growth and proliferation of HUVECs on different dressings. Figure 7A illustrated the CCK-8 assay absorbance of cells cultured on CAF nonwovens, composite dressings with $4 \%(w / v)$ CM-Chit and composite dressings with $10 \%(w / v)$ Mino. With the increase of incubation time, the numbers of cells cultured on the three dressings increased significantly, showing that the fabricated dressings possessed better biocompatibility. The absorbance of cells cultured on the CM-Chit gel surface of the composite dressings was slightly higher than that of cells cultured on CAF nonwovens, which indicated that the composite dressings had better biocompatibility. Moreover, with the increase of CM-Chit concentration, the absorbance of cells cultured on the gel surface increased slightly (Figure 7B). The previous research showed that Mino had no toxicity to cells [51]. The absorbance of cells cultured on the surface of the Mino loaded composite dressings was not significantly different from that of cells cultured on the surface of the composite dressings. Therefore, the loading of Mino did not affect the growth and proliferation of cells on the surface of the composite dressings.

Figure 7C showed stained live cells CLSM images to characterize the morphology and spreading of cells cultured on the dressings. The cells attached to the dressings with good morphology. Due to the proliferation and spreading of cells, the amount and the distribution range of cells increased significantly over the incubation time. Compared with the rough surface structure of CAF nonwovens, the smooth CM-Chit gel structure of the composite dressings provided better spreading conditions for cells. Therefore, cells cultured on CM-Chit gel surface of the composite dressings had better growth and proliferation activity. 

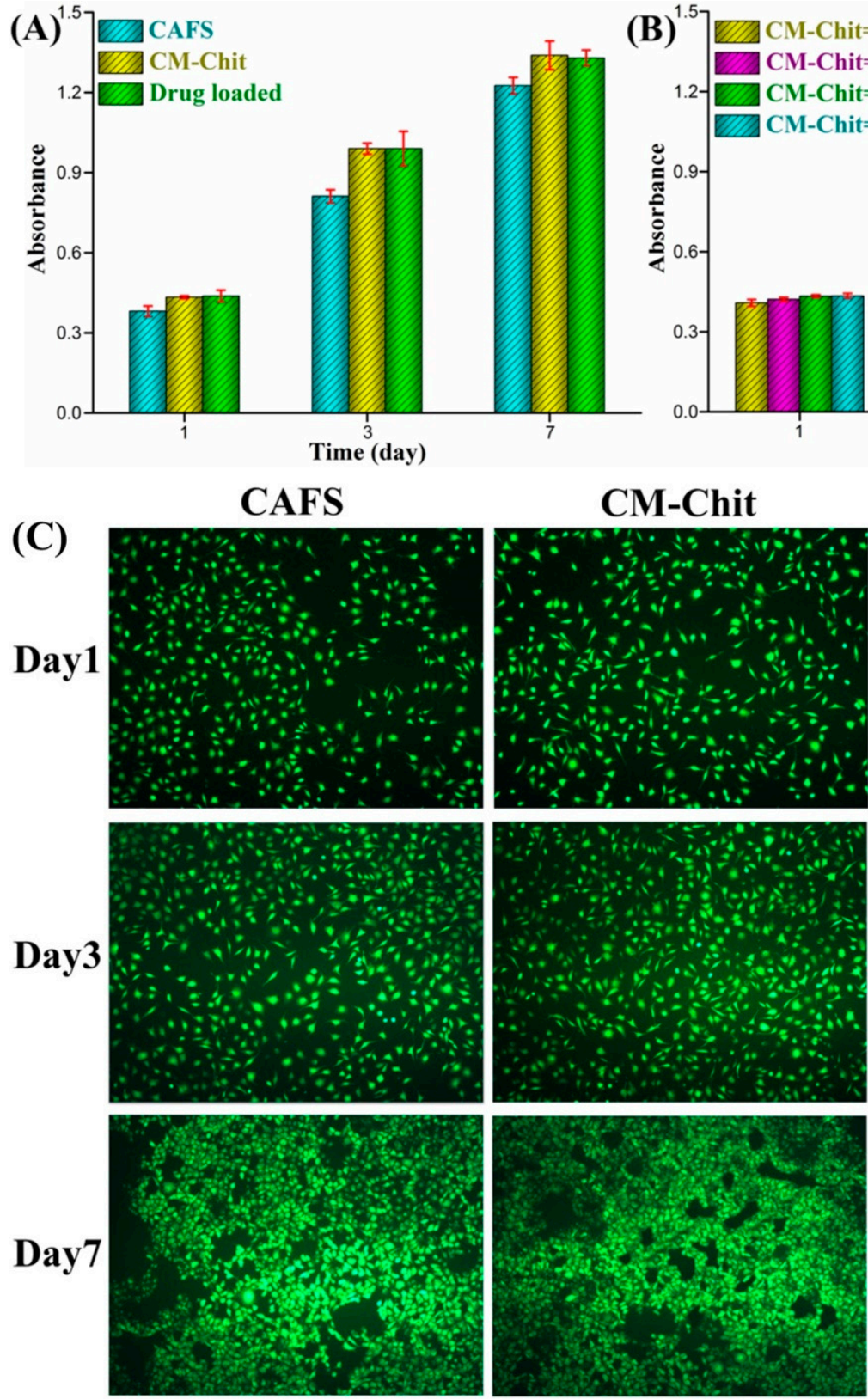

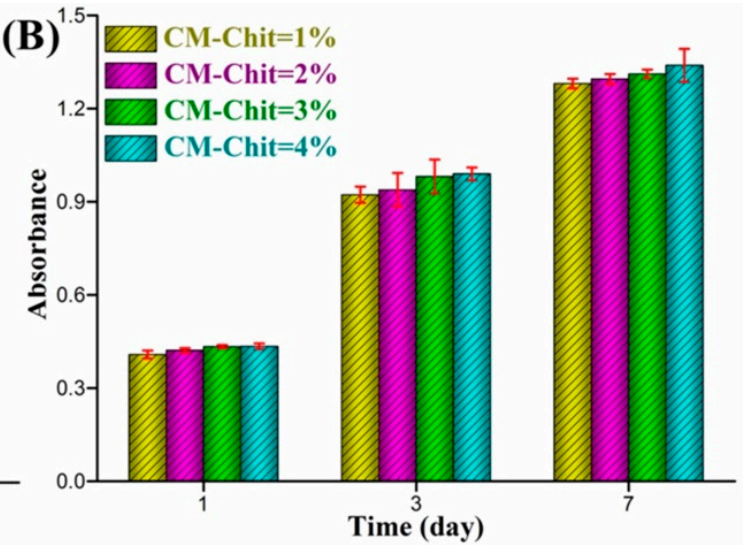

Drug loaded
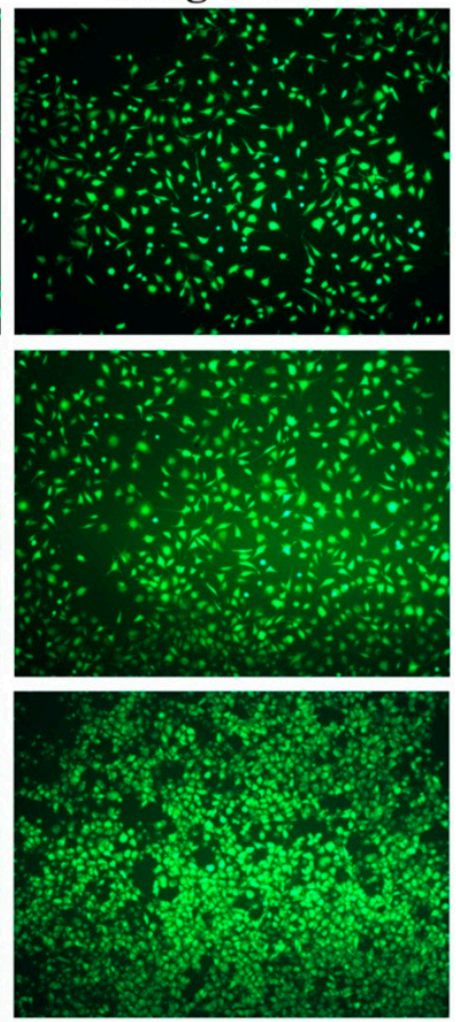

Figure 7. Cell Cytotoxicity of the composite wound dressings: (A) The proliferation of cells cultured on different dressings. (B) The proliferation of cells cultured on the CM-Chit gel surface. (C) The confocal laser scanning microscope (CLSM) images of the cells cultured on the CAF nonwovens, CM-Chit gel surface of the composite dressings and the drug loaded composite dressings.

\section{Materials and Methods}

\subsection{Materials}

The CAF (3.01 dtex $\pm 0.03 \mathrm{dtex}, 50 \mathrm{~mm}$ ) was provided from United Medical Technologies Co., Ltd. (Foshan, China). Carboxymethyl chitosan (CM-Chit) was purchased from Yuanye Bio-Technology Co., Ltd. (Shanghai, China). Its degree of deacetylation and degree of substitution were $85 \%$ and $80 \%$ respectively. 1-3-(3-dimethylaminopropyl)-3-ethylcarbodiimide hydrochloride (EDC), $\mathrm{N}$-hydroxysuccinimide (NHS) and minocycline hydrochloride were purchased from Sigma-Aldrich (St. Louis, MO, USA). Ethyl alcohol and sodium chloride were purchased from Aladdin (Shanghai, China). Reagents used in bacterial culture assay (yeast extract, tryptone, agar, etc.) were obtained from 
Thermo Scientific Oxoid (Basingstoke, UK). Reagents used in cell culture in vitro were purchased from Gibco (Thermo Fisher Scientific Co., Waltham, MA, USA). Cell Counting Kit (CCK8) was purchased from Gibco (Thermo Fisher Scientific Co., Waltham, MA, USA). Cell viability (Calcein-AM/PI) test kit was procured from KeyGEN BioTECH Co. (Nanjing, China). The water purified by a Milli-Q water purification system (Millipore, Bedford, MA, USA) was used in all the experiments and the resistivity higher than $18.2 \mathrm{M} \Omega$.

\subsection{Fabrication of CM-Chit Gel/CAF Nonwovens Composite Wound Dressings}

As shown in Figure 8, the process of fabrication for CM-Chit gel/CAF nonwovens composite wound dressings was divided into four steps. Step 1: Fabrication of CAF nonwovens. Calcium alginate fibers were opened and carded into fiber webs and then the webs were cross-lapped and needle-punched, forming the needle-punched nonwovens with a porous 3D structure and a surface density of $120 \mathrm{~g} / \mathrm{m}^{2}$. Step 2: CM-Chit solution preparation and plasma treatment of CAF nonwovens. $\mathrm{CM}$-Chit was dissolved in Milli-Q water and stirred to form a uniform solution. The fluidity and surface tension of CM-Chit solution determined the diffusion of the solution in nonwovens. The rheological behaviors of CM-Chit solution were measured by ARES rheometers (TA Instruments Co., New Castle, DE, USA). The surface tension of CM-Chit solution was measured and characterized by the surface tensiometer (DCAT11, Data Physics Instruments Co., Filderstadt, Germany). In order to improve the affinity of CAF nonwovens and the diffusion of CM-Chit solution in nonwovens, surface oxygen plasma treatment on CAF nonwovens was carried out by low-temperature plasma treatment instrument (HD-300, Zhongke ChangTsi Plasma Processing Apparatus Plasma Technology Co., Changzhou, China), the treatment time was set as 20 s. Step 3: CM-Chit solution coating. CM-Chit solution was coated on the surface of CAF nonwovens using a small laboratory coating machine (MSK-AFA-III, Milliren Technologies, Inc., Newburyport, MA, USA). The running speed of the scraper was set as $15 \mathrm{~mm} / \mathrm{s}$, and the distance between the scraper and the surface of CAF nonwovens was set as $1 \mathrm{~mm}$. Step 4: CM-Chit crosslinking. CAF nonwovens coated with CM-Chit solution was immersed in EDC/NHS solution ( $90 \%$ ethanol and $10 \%$ water) and placed at room temperature for crosslinking. After $24 \mathrm{~h}$, the samples were taken out and washed with Milli-Q water for 3 times to remove excess EDC/NHS and ethanol.
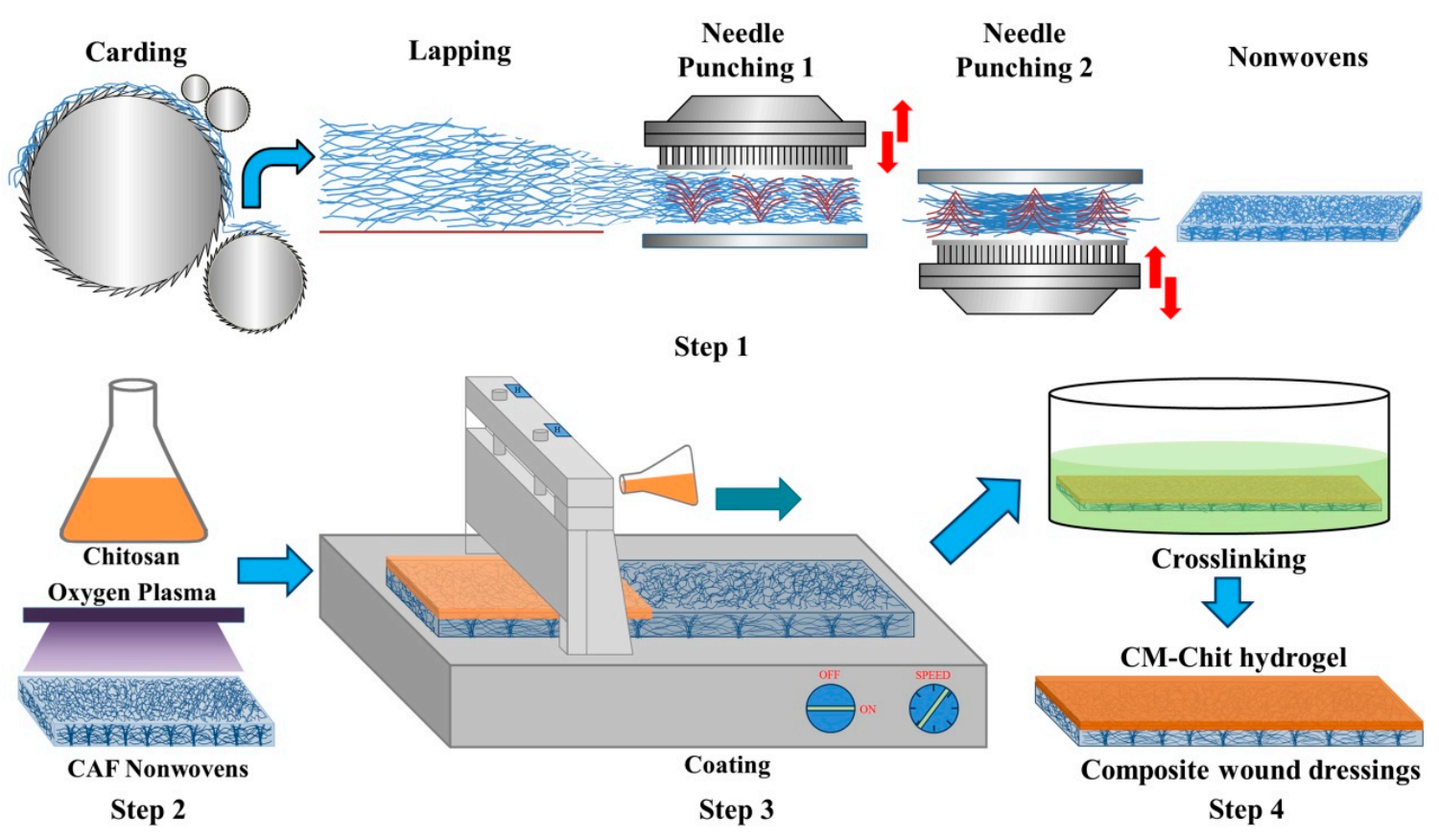

Figure 8. Schematic diagram of fabrication process of CM-Chit gel/CAF nonwovens composite wound dressings. 


\subsection{Morphology and Porous Structure Characterization}

The surface and cross-section morphology of the lyophilized composite wound dressings were observed by a scanning electron microscope (TM3000, Hitachi, Tokyo, Japan) at an acceleration voltage of $10 \mathrm{kV}$. The porous structure of composite wound dressings, including the pore size and it distribution, was measured using the capillary flow porosimetry (CFP, Porometer $3 \mathrm{G} \mathrm{zh}$; Quantachrome Instruments, Boynton Beach, FL, USA).

\subsection{Macromolecular Structure Characterization}

The macromolecular structure of composite wound dressings was characterized by Fourier Transform Infrared (FTIR) and X-ray diffraction (XRD). The FTIR spectra were measured by Nicolet 6700 FTIR spectroscopy (Thermo Fisher Scientific, Waltham, MA, USA) in the attenuated total reflection mode (ATR). The absorption spectral range was $500-4000 \mathrm{~cm}^{-1}$. The crystal and aggregation structure of CM-Chit and CAF macromolecules were investigated by the D/max RB X-ray diffractometer (Rigaku Co., Tokyo, Japan) with Nickel-filtered $\mathrm{Cu} k \alpha$ radiation in the $2 \theta$ range of $0-60^{\circ}$.

\subsection{Solution Absorption Performance Testing}

The hydrophilicity of the composite wound dressings can be characterized by the contact angle. The contact angles the different surfaces of composite wound dressings were measured by the Contact Angle Tester (OCA15EC; Data Physics Instruments Co., Filderstadt, Germany). The samples ( $5 \mathrm{~cm} \times$ $5 \mathrm{~cm}$, weight as $W_{0}$ ) were immersed in Milli-Q water at $37^{\circ} \mathrm{C}$ for $0.5,1,2,4,6,8,12,24 \mathrm{~h}$. The samples after absorption of water were weighed after removing the residual water $\left(W_{1}\right)$. The solution uptake rate $(g / g)$ was calculated by $\left(W_{1}-W_{0}\right) / W_{0}$.

\subsection{Tensile Mechanical Performance Testing}

The tensile mechanical performance of the composite wound dressings was measured by an electromechanical universal testing machine (WDW-20; Shanghai Hualong Test Instruments Co., Shanghai, China) with a gauge length of $10 \mathrm{~cm}$ and a stretching speed of $50 \mathrm{~mm} / \mathrm{min}$.

\subsection{Mino Loading and in Vitro Release}

\subsubsection{Fabrication of Drug Loaded CM-Chit gel/CAF Nonwovens Composite Wound Dressings}

For drug loading, Mino was dissolved to CM-Chit solution and the mixture was stirred at room temperature in dark conditions to form uniform Mino/CM-Chit solution, and then Mino loaded CM-Chit gel/CAF nonwovens composite wound dressings were fabricated as described in Section 3.2 in dark conditions. The crosslinking solution and washing solution were collected and measured by a UV spectrophotometer (JASCO V530, JASCO, Tokyo, Japan) at $272 \mathrm{~nm}$. The Mino loading content (Lc) was calculated according to Equation (1):

$$
\text { Loading content }=\left(1-\frac{C \times V}{m}\right) \times 100 \%
$$

Where $C$ was the drug concentration of the collected solution; $V$ was the volume of the collected solution; $m$ was the weight of Mino in CM-Chit solution.

\subsubsection{In Vitro Mino Release}

The drug release from the Mino loaded CM-Chit gel/CAF nonwovens composite wound dressings was investigated in accordance with the methods described in the previous research [52]. A total of $1 \mathrm{~g}$ of each sample was immersed in centrifuge tube with $100 \mathrm{~mL}$ PBS solution $(\mathrm{pH}=7.4)$ and placed in a shaker with a constant temperature of $37^{\circ} \mathrm{C}$ and a speed of $100 \mathrm{rpm}$. After the selected time interval of release, $5 \mathrm{~mL}$ of the released solution was taken out and measured by a UV spectrophotometer 
at $272 \mathrm{~nm}$. In order to ensure the uniformity of the release solution, $5 \mathrm{~mL}$ fresh PBS solution was added after each extraction. The concentration of the released solution was calculated according to the standard calibration of Mion in PBS solution. The cumulative release ratio of Mino was calculated according to Equation (2):

$$
\mathrm{Q}(\%)=\frac{C_{n} \cdot V+V_{i} \sum_{i=0}^{n-i} C_{i}}{m_{d}}
$$

where, $\mathrm{Q}$ was the cumulative release ratio of Mino; $n$ was number of displacement release solution; $\mathrm{Cn}$ was the concentration of drug in the release solution at nth release time interval, $\mathrm{mg} / \mathrm{mL} ; V$ was the volume of the release solution; $V i$ was the volume of drug release solution at the ith release time interval; $C i$ was the concentration of drug in the release solution at ith release time interval, $\mathrm{mg} / \mathrm{mL} ; V_{0}$ and $C_{0}$ were $0 ; m_{d}$ was the quality of drug in sample.

\subsection{In Vitro Antibacterial Activity Assay}

The antibacterial activity of the fabricated wound dressings was evaluated by the agar plate diffusion method. Escherichia coli (E. coli), a gram-positive bacterium, and Staphylococcus aureus (S. aureus), a gram-positive bacterium, were used in this assay. The frozen E. coli and S. aureus were activated and cultured in the trypticase soy broth (TSB) medium in an oscillation incubator with a temperature of $37^{\circ} \mathrm{C}$ and a speed of $120 \mathrm{rpm}$. The bacterium/TSB suspension was spread on the agar plates and the sterilized circular samples $(\mathrm{n}=3)$ with a diameter of $14 \mathrm{~mm}$ were placed on the spread bacterium/TSB suspension. The agar plates were incubated in a constant temperature incubator with a temperature of $37^{\circ} \mathrm{C}$. After $24 \mathrm{~h}$, the diameter of the inhibition zone was calculated from the images of agar plates captured by the automatic colony counter (Hangzhou Shineso Science \& Technology, Hangzhou, China). The width of inhibition zone (H) was calculated according to Equation (3):

$$
\mathrm{H}=\frac{\text { Outer diameter of the inhibition zone }-14 \mathrm{~mm}}{2}
$$

The Mino loaded wound dressings samples were placed in PBS for drug release. After 1, 3, 7 days, the antimicrobial activity of the samples was measured using the same method.

\subsection{In Vitro Cell Cytotoxicity Assay}

\subsubsection{Cell Culture}

Human umbilical vein endothelial cells (HUVECs, Cell Bank of the Chinese Academy of Science, Shanghai, China) were cultured in medium with $90 \%(v / v)$ high glucose DMEM, $10 \%(v / v)$ fetal bovine serum (FBS) and $1 \%(v / v)$ penicillin/streptomycin at $37^{\circ} \mathrm{C}$ and $5 \% \mathrm{CO}_{2}$. The sterilized wound dressings were placed in 24 -well cell culture plates. The HUVECs $\left(10^{5}\right.$ cells/well $)$ were seeded on the samples and cultured in medium at $37{ }^{\circ} \mathrm{C}$ and $5 \% \mathrm{CO}_{2}$.

\subsubsection{Cell Proliferation Assay}

The proliferation of cells on the wound dressings was measured by CCK- 8 assay. After 1, 3 and 7 days of culture, the medium was removed and $400 \mu \mathrm{L}$ CCK- 8 test solution was added into the well and cultured in dark conditions at $37^{\circ} \mathrm{C}$ and $5 \% \mathrm{CO}_{2}$. After $4 \mathrm{~h}$ of incubation, the absorbance value (O.D. Values) at $450 \mathrm{~nm}$ of the supernatant in each well was measured by a microplate reader (Multiskan GO, Thermo, Waltham, MA, USA).

\subsubsection{Cell Morphology}

After 1, 3 and 7 days of culture, the medium was removed and the cells were washed by PBS for 3 times. Calcein-AM working solution was added to stain the cells and incubated at $37^{\circ} \mathrm{C}$ and $5 \%$ 
$\mathrm{CO}_{2}$. After $1 \mathrm{~h}$, the working solution was removed and the stained cells were washed by PBS and then visualized by confocal laser scanning microscope (CLSM, LSM 700, Carl Zeiss, Oberkochen, Germany).

\section{Conclusions}

In this study, minocycline/carboxymethyl chitosan solution was coated on the surface of plasma treated calcium alginate fibers needle-punched nonwovens and then immersed in EDC/NHS crosslinking solution. CM-Chit was crosslinked to form gel, thereby Mino loaded CM-Chit gel/CAF nonwovens composite dressings were fabricated. SEM images showed that the composite dressings had a porous structure, which was composed of gel, gel/nonwovens composite structure and nonwovens porous structure. With the increase of CM-Chit solution concentration, the thickness of gel structure and the pore size distribution was changed. The dressings had excellent absorption performance and quickly reached the maximum absorption volume so that wound exudates could be quickly transferred from the wound. The excellent mechanical properties of CAF nonwovens greatly improved the mechanical properties of CM-Chit gel so that the composite dressings had similar mechanical properties to skin. The addition of Mino significantly increased the antimicrobial activity of the composite dressings, and the gel structure avoided the burst release of the drug, which enabled the drug in the effective concentration within a certain period of time to achieve the antibacterial effect. The cells cultured on the composite dressings had good growth and proliferation activity. As new functional dressings, due to the excellent absorption performance, skin-like mechanical properties, long-term antimicrobial activity, drug sustained-release properties and biocompatibility, the prepared composite dressings had great potential in wound healing.

Author Contributions: Y.G. and X.J. designed the experiments; Y.G. and X.Z. performed the experiments; Y.G. and X.J. analyzed the data; Y.G. wrote the manuscript; Y.G., X.Z., Y.W. and X.J. revised the manuscript. All authors contributed to the research and the manuscript.

Funding: This research was funded by the Fundamental Research Funds for the Central Universities from Donghua University, grant number CUSF-DH-D-2016018.

Acknowledgments: We thank the support of Yuxiao Wang in the final revision work.

Conflicts of Interest: The authors declare no conflict of interest.

\section{References}

1. Thomas, S. Alginate dressings in surgery and wound management-Part 1. J. Wound Care 2000, 9, 56-60. [CrossRef] [PubMed]

2. Powers, J.G.; Morton, L.M.; Phillips, T.J. Dressings for chronic wounds. Dermatol. Ther. 2013, 26, $197-206$. [CrossRef] [PubMed]

3. Yue, M.; Lei, M.; Liu, Y.; Gui, N. The application of moist dressings in wound care for tracheostomy patients: A meta-analysis. J. Clin. Nurs. 2019, 28, 2724-2731. [CrossRef] [PubMed]

4. Gaspar-Pintiliescu, A.; Stanciuc, A.M.; Craciunescu, O. Natural composite dressings based on collagen, gelatin and plant bioactive compounds for wound healing: A review. Int. J. Biol. Macromol. 2019, 138, 854-865. [CrossRef] [PubMed]

5. Rezvani Ghomi, E.; Khalili, S.; Nouri Khorasani, S.; Esmaeely Neisiany, R.; Ramakrishna, S. Wound dressings: Current advances and future directions. J. Appl. Polym. Sci. 2019, 136, 47738. [CrossRef]

6. Rosenbaum, A.J.; Banerjee, S.; Rezak, K.M.; Uhl, R.L. Advances in wound management. J. Am. Acad. Orthop. Surg. 2018, 26, 833-843. [CrossRef] [PubMed]

7. Jaffe, L.; Wu, S.C. Dressings, topical therapy, and negative pressure wound therapy. Clin. Podiatr. Med. Sur. 2019, 36, 397-411. [CrossRef]

8. Öhnstedt, E.; Lofton Tomenius, H.; Vågesjö, E.; Phillipson, M. The discovery and development of topical medicines for wound healing. Expert Opin. Drug Dis. 2019, 14, 485-497. [CrossRef]

9. Struszczyk, M.H. Chitin and chitosan: Part Iproperties and production. Polimery 2002, 47, 316-325. [CrossRef]

10. Struszczyk, M.H. Chitin and chitosan: Part II applications of chitosan. Polimery 2002, 47, 396-403. [CrossRef] 
11. Muzzarelli, R.A.A. Chitins and chitosans for the repair of wounded skin, nerve, cartilage and bone. Carbohydr. Polym. 2009, 76, 167-182. [CrossRef]

12. Ahsan, S.M.; Thomas, M.; Reddy, K.K.; Sooraparaju, S.G.; Asthana, A.; Bhatnagar, I. Chitosan as biomaterial in drug delivery and tissue engineering. Int. J. Biol. Macromol. 2018, 110, 97-109. [CrossRef] [PubMed]

13. Patrulea, V.; Ostafe, V.; Borchard, G.; Jordan, O. Chitosan as a starting material for wound healing applications. Eur. J. Pharm. Biopharm. 2015, 97, 417-426. [CrossRef] [PubMed]

14. Kumar, M.N.V.R. A review of chitin and chitosan applications. React. Funct. Polym. 2000, 46, 1-27. [CrossRef]

15. Laurienzo, P. Marine polysaccharides in pharmaceutical applications: An overview. Mar. Drugs 2010, 8, 2435-2465. [CrossRef] [PubMed]

16. Dragostin, O.M.; Samal, S.K.; Dash, M.; Lupascu, F.; Pânzariu, A.; Tuchilus, C.; Ghetu, N.; Danciu, M.; Dubruel, P.; Pieptu, D.; et al. New antimicrobial chitosan derivatives for wound dressing applications. Carbohydr. Polym. 2016, 141, 28-40. [CrossRef]

17. Baldrick, P. The safety of chitosan as a pharmaceutical excipient. Regul. Toxicol. Pharmacol. 2010, 56, $290-299$. [CrossRef] [PubMed]

18. Kojima, K.; Okamoto, Y.; Kojima, K.; Miyatake, K.; Fujise, H.; Shigemasa, Y.; Minami, S. Effects of chitin and chitosan on collagen synthesis in wound healing. J. Vet. Med. Sci. 2004, 66, 1595-1598. [CrossRef]

19. Azad, A.K.; Sermsintham, N.; Chandrkrachang, S.; Stevens, W.F. Chitosan membrane as a wound-healing dressing: Characterization and clinical application. J. Biomed. Mater. Res. B Appl Biomater. 2004, 69B, $216-222$. [CrossRef]

20. Kong, M.; Chen, X.G.; Xing, K.; Park, H.J. Antimicrobial properties of chitosan and mode of action: A state of the art review. Int. J. Food Microbiol. 2010, 144, 51-63. [CrossRef]

21. Choi, C.; Nam, J.P.; Nah, J.W. Application of chitosan and chitosan derivatives as biomaterials. J. Ind. Eng. Chem. 2016, 33, 1-10. [CrossRef]

22. Takei, T.; Danjo, S.; Sakoguchi, S.; Tanaka, S.; Yoshinaga, T.; Nishimata, H.; Yoshida, M. Autoclavable physically-crosslinked chitosan cryogel as a wound dressing. J. Biosci. Bioeng. 2018, 125, 490-495. [CrossRef] [PubMed]

23. Bukzem, A.L.; Signini, R.; Dos Santos, D.M.; Lião, L.M.; Ascheri, D.P. Optimization of carboxymethyl chitosan synthesis using response surface methodology and desirability function. Int. J. Biol. Macromol. 2016, 85, 615-624. [CrossRef] [PubMed]

24. Faizuloev, E.; Marova, A.; Nikonova, A.; Volkova, I.; Gorshkova, M.; Izumrudov, V. Water-soluble $\mathrm{N}$-[(2-hydroxy-3-trimethylammonium)propyl]chitosan chloride as a nucleic acids vector for cell transfection. Carbohydr. Polym. 2012, 89, 1088-1094. [CrossRef] [PubMed]

25. Chivangkul, T.; Pengprecha, S.; Padungros, P.; Siraleartmukul, K.; Prasongsuk, S.; Muangsin, N. Enhanced water-solubility and mucoadhesion of N, N, N-trimethyl-N-gluconate-N-homocysteine thiolactone chitosan. Carbohydr. Polym. 2014, 108, 224-231. [CrossRef] [PubMed]

26. Upadhyaya, L.; Singh, J.; Agarwal, V.; Ravi Prakash, T. Biomedical applications of carboxymethyl chitosans. Carbohydr. Polym. 2013, 91, 452-466. [CrossRef] [PubMed]

27. Lin, Y.-H.; Liang, H.-F.; Chung, C.-K.; Chen, M.-C.; Sung, H.-W. Physically crosslinked alginate/N,O-carboxymethyl chitosan hydrogels with calcium for oral delivery of protein drugs. Biomaterials 2005, 26, 2105-2113. [CrossRef] [PubMed]

28. Xu, W.; Liu, K.; Li, T.; Zhang, W.; Dong, Y.; Lv, J.; Wang, W.; Sun, J.; Li, M.; Wang, M.; et al. An in situ hydrogel based on carboxymethyl chitosan and sodium alginate dialdehyde for corneal wound healing after alkali burn. J. Biomed. Mater. Res. A 2019, 107, 742-754. [CrossRef] [PubMed]

29. Shi, X.; Du, Y.; Sun, L.; Yang, J.; Wang, X.; Su, X. Ionically crosslinked alginate/carboxymethyl chitin beads for oral delivery of protein drugs. Macromol. Biosci. 2005, 5, 881-889. [CrossRef] [PubMed]

30. Zhu, A.; Chen, J.; Liu, Q.; Jiang, Y. Controlled release of berberine hydrochloride from alginate microspheres embedded within carboxymethyl chitosan hydrogels. J. Appl. Polym. Sci. 2011, 120, 2374-2380. [CrossRef]

31. Liu, X.; Guan, Y.; Yang, D.; Li, Z.; Yao, K. Antibacterial action of chitosan and carboxymethylated chitosan. J. Appl. Poly. Sci. 2001, 79, 1324-1335.

32. Upadhyaya, L.; Singh, J.; Agarwal, V.; Tewarim, R.P. The implications of recent advances in carboxymethyl chitosan based targeted drug delivery and tissue engineering applications. J. Control. Release 2014, 186, 54-87. [CrossRef] [PubMed] 
33. Shariatinia, Z. Carboxymethyl chitosan: Properties and biomedical applications. Int. J. Biol. Macromol. 2018, 120, 1406-1419. [CrossRef]

34. Anitha, A.; Divya Rani, V.V.; Krishna, R.; Sreeja, V.; Selvamurugan, N.; Nair, S.V.; Tamura, H.; Jayakumar, R. Synthesis, characterization, cytotoxicity and antibacterial studies of chitosan, O-carboxymethyl and N,O-carboxymethyl chitosan nanoparticles. Carbohydr. Polym. 2009, 78, 672-677. [CrossRef]

35. Lee, K.Y.; Mooney, D.J. Alginate: Properties and biomedical applications. Prog. Polym. Sci. 2012, 37, $06-126$. [CrossRef]

36. Sikorski, P.; Mo, F.; Skjåk-Bræk, G.; Stokke, B.T. Evidence for egg-box-compatible interactions in calcium-alginate gels from fiber x-ray diffraction. Biomacromolecules 2007, 8, 2098-2103. [CrossRef]

37. Braccini, I.; Perez, S. Molecular basis of $\mathrm{Ca}^{2+}$-induced gelation in alginates and pectins: The egg-box model revisited. Biomacromolecules 2001, 2, 1089-1096. [CrossRef]

38. Rowley, J.A.; Madlambayan, G.; Mooney, D.J. Alginate hydrogels as synthetic extracellular matrix materials. Biomaterials 1999, 20, 45-53. [CrossRef]

39. Tellechea, A.; Silva, E.A.; Min, J.H.; Leal, E.C.; Auster, M.E.; Nabzdyk, L.P.; Shih, W.; Mooney, D.J.; Aristidis, V. Alginate and DNA gels are suitable delivery systems for diabetic wound healing. Int. J. Low. Extrem. Wounds 2015, 14, 146-153. [CrossRef]

40. Tønnesen, H.H.; Karlsen, J. Alginate in drug delivery systems. Drug Dev. Ind. Pharm. 2002, 28, 621-630. [CrossRef]

41. Schmitt, A.; Rödel, P.; Anamur, C.; Seeliger, C.; Imhoff, A.B.; Herbst, E.; Vogt, S.; Griensven, M.; Winter, G.; Engert, J. Calcium alginate gels as stem cell matrix-making paracrine stem cell activity available for enhanced healing after surgery. PLoS ONE 2015, 10, e0118937. [CrossRef] [PubMed]

42. Barralet, J.E.; Wang, L.; Lawson, M.; Triffitt, J.T.; Cooper, P.R.; Shelton, R.M. Comparison of bone marrow cell growth on 2D and 3D alginate hydrogels. J. Mater. Sci. Mater. Med. 2005, 16, 515-519. [CrossRef] [PubMed]

43. Paul, W.; Sharma, C.P. Chitosan and alginate wound dressings: A short review. Trends Biomater. Artif. Organs. 2004, 18, 18-23.

44. Dumville, J.C.; O’Meara, S.; Deshpande, S.; Speak, K. Alginate dressings for healing diabetic foot ulcers. Cochrane Database Syst. Rev. 2013, 6, 1-55. [CrossRef]

45. Ng, R.W.; Cheng, Y.L. Calcium alginate dressing-related hypercalcemia. J. Brun Care Res. 2007, 28, $203-204$. [CrossRef]

46. Tavakol, M.; Vasheghani-Farahani, E.; Dolatabadi-Farahani, T.; Hashemi-Najafabadi, S. Sulfasalazine release from alginate-N, O-carboxymethyl chitosan gel beads coated by chitosan. Carbohydr. Polym. 2009, 77, 326-330. [CrossRef]

47. Hu, Y.; Zhang, Z.; Li, Y.; Ding, X.; Li, D.; Chen, C.; Xu, F. Dual-crosslinked amorphous polysaccharide hydrogels based on Chitosan/Alginate for wound healing applications. Macromol. Rapid Comm. 2018, 39, 1800069. [CrossRef]

48. Bormashenko, E. Contact angles of sessile droplets deposited on rough and flat surfaces in the presence of external fields. Math. Model Nat. Phenom. 2012, 7, 1-5. [CrossRef]

49. Zahiri, B.; Sow, P.K.; Kung, C.H.; Mérida, W. Understanding the wettability of rough surfaces using simultaneous optical and electrochemical analysis of sessile droplets. J. Colloid Interface Sci. 2017, 501, 34-44. [CrossRef]

50. Chiarini, A.; Freddi, G.; Lium, D.; Armato, U.; Dal Prà, I. Biocompatible silk noil-based three-dimensional carded-needled nonwoven scaffolds guide the engineering of novel skin connective tissue. Tissue Eng. A 2016, 22, 1047-1060. [CrossRef]

51. Sung, J.H.; Hwang, M.R.; Kim, J.O.; Lee, J.H.; Kim, Y.; Kim, J.H.; Chang, S.W.; Jin, S.G.; Kim, J.A.; Lyoo, W.S.; et al. Gel characterisation and in vivo evaluation of minocycline-loaded wound dressing with enhanced wound healing using polyvinyl alcohol and chitosan. Int. J. Pharm. 2010, 392, 232-240. [CrossRef] [PubMed]

52. Zhou, X.; Weng, W.; Chen, B.; Feng, W.; Wang, W.; Nie, W.; Chen, L.; Mo, X.; Su, J.; He, C. Mesoporous silica nanoparticles/gelatin porous composite scaffolds with localized and sustained release of vancomycin for treatment of infected bone defects. J. Mater. Chem. B 2018, 6, 740-752. [CrossRef] 